\title{
The Neural Correlates of Motor Skill Automaticity
}

\author{
Russell A. Poldrack, ${ }^{1,3,4}$ Fred W. Sabb, ${ }^{4,5}$ Karin Foerde, ${ }^{1}$ Sabrina M. Tom, ${ }^{1}$ Robert F. Asarnow, ${ }^{2}$ \\ Susan Y. Bookheimer, ${ }^{2,3,4,5}$ and Barbara J. Knowlton ${ }^{1,3,4}$ \\ Departments of ${ }^{1}$ Psychology and ${ }^{2}$ Psychiatry, ${ }^{3}$ Brain Research Institute, ${ }^{4}$ Interdepartmental Neuroscience Program, and ${ }^{5}$ Brain Mapping Center, University \\ of California, Los Angeles, Los Angeles, California 90095
}

Acquisition of a new skill is generally associated with a decrease in the need for effortful control over performance, leading to the development of automaticity. Automaticity by definition has been achieved when performance of a primary task is minimally affected by other ongoing tasks. The neural basis of automaticity was examined by testing subjects in a serial reaction time (SRT) task under both single-task and dual-task conditions. The diminishing cost of dual-task performance was used as an index for automaticity. Subjects performed the SRT task during two functional magnetic imaging sessions separated by $3 \mathrm{~h}$ of behavioral training over multiple days. Behavioral data showed that, by the end of testing, subjects had automated performance of the SRT task. Before behavioral training, performance of the SRT task concurrently with the secondary task elicited activation in a wide network of frontal and striatal regions, as well as parietal lobe. After extensive behavioral training, dual-task performance showed comparatively less activity in bilateral ventral premotor regions, right middle frontal gyrus, and right caudate body; activity in other prefrontal and striatal regions decreased equally for single-task and dual-task conditions. These data suggest that lateral and dorsolateral prefrontal regions, and their corresponding striatal targets, subserve the executive processes involved in novice dual-task performance. The results also showed that supplementary motor area and putamen/globus pallidus regions showed training-related decreases for sequence conditions but not for random conditions, confirming the role of these regions in the representation of learned motor sequences.

Key words: basal ganglia; learning; prefrontal; executive control; automaticity; sequence learning

\section{Introduction}

A universal feature of human behavior is that well practiced tasks can be performed with relatively little effort or cognitive control, whereas novice performance of the same task may require intense and effortful cognitive control (James, 1890). The ability to perform skilled tasks without the need for executive control is referred to as "automaticity," and it has been extensively investigated by cognitive scientists over the past 40 years. A defining characteristic of automaticity is the ability to perform the automatized task with little or no interference by a demanding secondary task (Posner and Snyder, 1975; Logan, 1979). The neural basis of automaticity is of great interest, but little is currently known about how neural activity changes as a task becomes automatized (but see Wu et al., 2004).

In the present study, we trained subjects extensively on an implicit sequential reaction time (SRT) task that has been used both behaviorally and with neuroimaging to examine motor skill

\footnotetext{
Received Sept. 18, 2004; revised March 20, 2005; accepted April 13, 2005.

This material is based on work supported under a National Science Foundation Graduate Research Fellowship (to K.F.). For generous support, we thank the Brain Mapping Medical Research Organization, Brain Mapping Support Foundation, Pierson-Lovelace Foundation, The Ahmanson Foundation, Tamkin Foundation, Jennifer Jones-Simon Foundation, Capital Group Companies Charitable Foundation, Robson Family, William M. and Linda R. Dietel Philanthropic Fund at the Northern Piedmont Community Foundation, Northstar Fund, and the National Center for Research Resources Grants RR12169, RR13642, and RR08655. We also thank Elizabeth 0'Hare for assistance with data analysis and Marie Luna for assistance with data collection.

Correspondence should be addressed to Russell A. Poldrack, Department of Psychology, University of California, Los Angeles, Franz Hall, Box 951563, Los Angeles, CA 90095-1563. E-mail: poldrack@ucla.edu.

DOI:10.1523/JNEUROSCI.3880-04.2005

Copyright $\odot 2005$ Society for Neuroscience $\quad$ 0270-6474/05/255356-09\$15.00/0
}

learning. In this paradigm (Nissen and Bullemer, 1987), subjects are presented with a four-alternative spatial choice reaction time task; unbeknownst to them, on some blocks of trials, the locations occur in a particular sequence, whereas on other blocks, the stimuli are presented in a pseudorandom order. Subjects will generally respond more quickly on all blocks as training progresses. In addition, there is a greater decrease in response time (RT) for sequence blocks compared with pseudorandom blocks, even if subjects cannot explicitly report the sequence (Willingham et al., 1989) (but see Perruchet and Amorim, 1992). We examined performance of the SRT task under both singletask and dual-task conditions. Subjects were scanned using functional magnetic resonance imaging (fMRI) on two occasions, with $3 \mathrm{~h}$ of SRT training in between these scanning sessions. Dual-task probe blocks were presented during both training and imaging, and degree of automaticity was defined as the cost of performing the secondary task.

The lateral prefrontal cortex has been particularly implicated in the support of cognitive control operations (Norman and Shallice, 1986; Miller and Cohen, 2001). In particular, there is evidence that lateral prefrontal regions are recruited under conditions of dual-task performance (Dreher and Grafman, 2003) and in situations requiring task switching (Sohn et al., 2000; Wylie et al., 2003). These results suggest that the development of automaticity should be associated with decreasing activity in lateral prefrontal regions. At the same time, previous studies of both motor (Grafton et al., 1994; Van Horn et al., 1998; Penhune and Doyon, 2002) and perceptual (Poldrack et al., 1998; Poldrack and Gabrieli, 2001) skill acquisition have found learning-related in- 


\section{A. Scanning run structure}

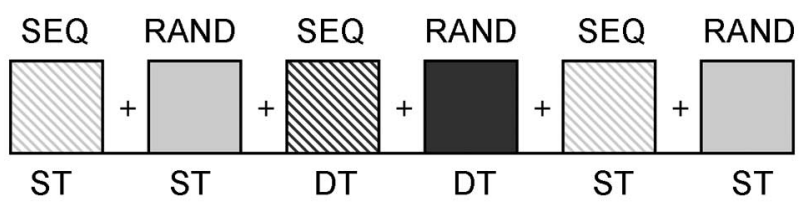

\section{B. SRT training session structure}

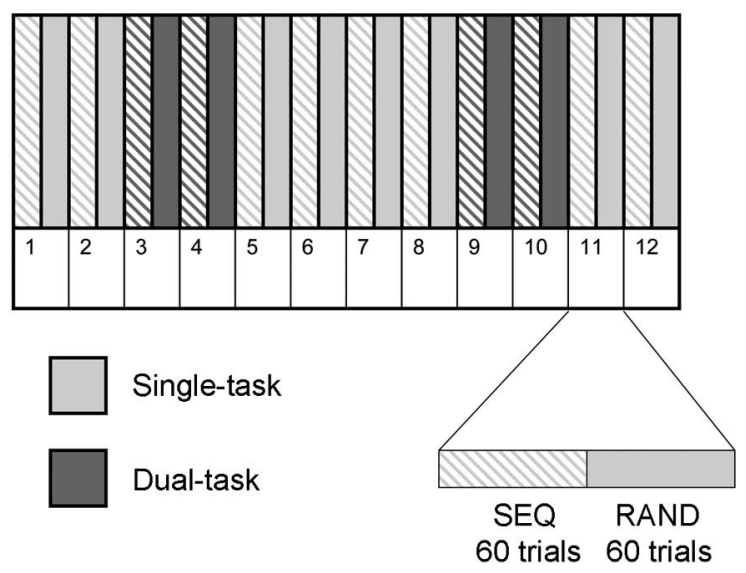

Figure 1. Schematic of the task design. $\boldsymbol{A}$, Blocked design used during fMRI scanning. ST, Single task; DT, dual task; SEQ, sequence; RAND, pseudorandom. The ordering of single-/dualtask blocks was constant for all subjects, whereas the order of sequence and random blocks was counterbalanced across runs and subjects. $\boldsymbol{B}$, Structure of the training sessions, showing alternation of sequence/pseudorandom blocks and placement of early/late dual-task probe blocks.

creases in activation in both cortical and subcortical regions, including the neostriatum (putamen for motor skill learning and caudate for perceptual skill learning). We tested for regions of either increased or decreased activation with automaticity to test the hypothesis that different regions may exhibit divergent changes related to the decreased need for cognitive control with practice.

\section{Materials and Methods}

Subjects. Fourteen normal young adult subjects participated in this study for payment (mean age, 20.7; eight females). All subjects provided informed consent according to procedures approved by the University of California, Los Angeles Human Subjects Committee. One additional subject was excluded because of failure to complete the second imaging session.

Experimental design. Each subject participated in two scanning sessions, separated by 1 or $2 \mathrm{~d}$. During the intervening period, subjects participated in three training sessions. Figure 1 presents a schematic of the task design.

The task was an SRT (Nissen and Bullemer, 1987), in which subjects were presented with a visual target in one of four screen locations and asked to respond as quickly and accurately as possible to this location by pressing one of four spatially compatible buttons. The visual target (" $\mathrm{X}$ ") was presented for $1 \mathrm{~s}$, with a $1.25 \mathrm{~s}$ interstimulus interval. During some blocks ("sequence" blocks), the stimulus locations followed a consistent 12-item-long sequence (unbeknownst to the subject). This was a secondorder conditional sequence, meaning that every individual location and every first-order transition appeared equally often. During other blocks (known as "pseudorandom" blocks), items were presented in a pseudorandom order, with the constraint that all individual targets appear equally often as in the sequence blocks (Reed and Johnson, 1994). This design ensures that any differences in behavior or activity between sequence and random blocks must reflect the learning of higher-order sequential knowledge rather than lower-order statistics.

Subjects performed the SRT task under both single-task and dual-task conditions. Under the single-task condition, only the SRT was performed. Under dual-task conditions, the SRT was performed concurrently with a tone-counting task. A single tone was presented each time a visual target appeared; across subjects, $50-70 \%$ of the tones were high pitched $(1000 \mathrm{~Hz})$, with the remaining tones at $500 \mathrm{~Hz}$. Subjects were asked to count the number of high tones occurring during each dual-task block; during scanning runs, they did not report these counts, whereas during training runs, they reported them by entering the number using a keyboard.

Each scanning session consisted of two functional imaging runs. During each of these runs, there were six blocks (lasting $45 \mathrm{~s}$ each), during which the subject performed the SRT task, separated by blocks of rest lasting $15 \mathrm{~s}$ each; each run also began with $15 \mathrm{~s}$ of rest. Each scan included the following: two single-task sequence blocks, two single-task pseudorandom blocks, one dual-task sequence block, and one dual-task pseudorandom block (Fig. 1). Two orders were used, with a different order for each of the two runs in each session (with order counterbalanced across runs and across sessions). Key-press responses and response times were collected using a custom four-button box.

During the intervening period between scanning sessions, subjects participated in three training sessions. During each session, they performed 12 blocks of the SRT task, each of which included 60 sequence trials and 60 pseudorandom trials. All blocks were performed under single-task conditions, except for blocks 3, 4, 9, and 10, which were performed under dual-task conditions as described above (Fig. 1).

Imaging procedures. Imaging was performed using a 3.0 T GE MR scanner with Advanced NMR gradients (GE Medical Systems, Milwaukee, WI). Blood oxygenation level-dependent-sensitive functional images were collected using a gradient-echo echo-planar pulse sequence [repetition time (TR), $3000 \mathrm{~ms}$; echo time (TE), $45 \mathrm{~ms} ; 64 \times 64$ matrix; 19 slices, $4 \mathrm{~mm}$ thick, $1 \mathrm{~mm}$ interslice gap]. Two runs of fMRI acquisition were performed for each subject during each scanning session, lasting $363 \mathrm{~s}$ each (encompassing $360 \mathrm{~s}$ for the task paradigm and an additional $3 \mathrm{~s}$ at the end of the run), with an additional four images at the beginning of the run discarded to allow T1 equilibration. Before functional scanning, we collected a coplanar anatomical image of the whole brain using a T2-weighted spin-echo echo-planar sequence to aid intersubject registration (TR, $4 \mathrm{~s}$; TE, $54 \mathrm{~ms}$; 128 matrix; 26 slices, $4 \mathrm{~mm}$ thick, $1 \mathrm{~mm}$ interslice gap).

$f M R I$ data analysis. Preprocessing and statistical analysis of the data were performed using SPM99 software (Wellcome Department of Cognitive Neurology, London, UK). The data were first analyzed for outlier images (caused by scanner spikes); such images were replaced with the mean of the two temporally adjacent images in the time series, with a total of 12 images replaced across the entire study. Preprocessing with SPM99 included motion correction and spatial normalization to a custom template approximating the MNI 305 stereotactic space (Montreal Neurological Institute), using linear affine registration followed by nonlinear registration using cosine basis functions, resampling to $3 \mathrm{~mm}$ cubic voxels. Normalization was performed by aligning the coplanar anatomical image to the template and then applying the same normalization parameters to the aligned functional images. Statistical analysis was first performed for each individual subject using a general linear model in SPM99. The design was modeled as a boxcar convolved with a canonical hemodynamic response, with each block modeled separately. Lowfrequency signal components (120 s cutoff) were treated as confounding covariates. The model fit was performed individually for each subject, and contrast images were generated for each of the four event types (against the explicitly unmodeled baseline/fixation). The contrast images were spatially smoothed with an $8 \mathrm{~mm}$ Gaussian kernel and then used in a second-level analysis treating subject as a random effect. The secondlevel statistical maps were thresholded at $p=0.005$ (uncorrected); control for familywise error was achieved using the cluster-level Gaussian random field results, resulting in a volumewise corrected error rate of $p<$ 0.05 . 

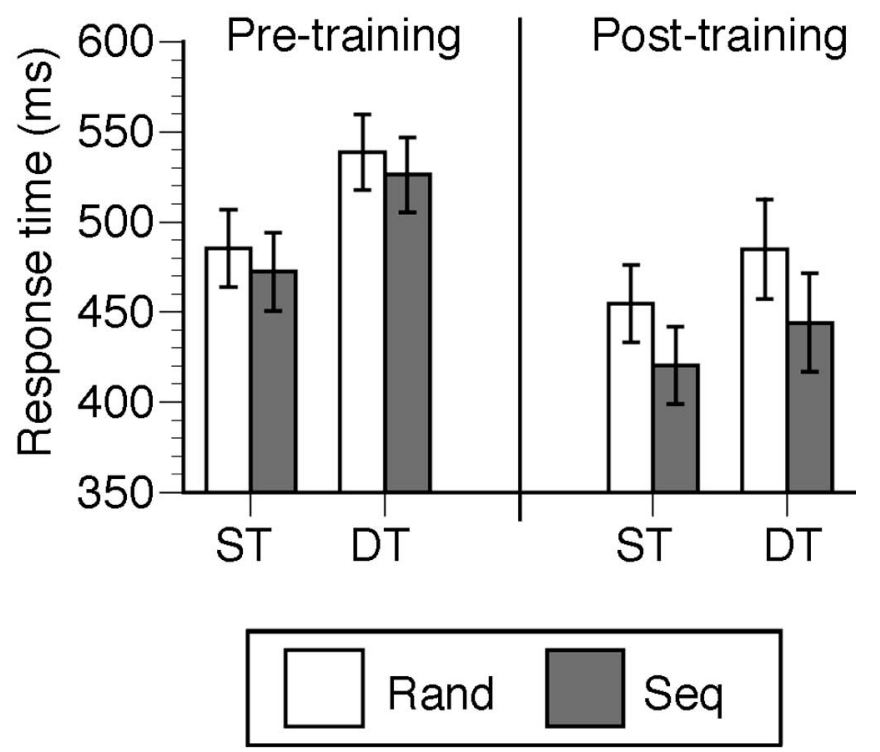

Figure 2. Response time data from fMRI scans, with SE bars. ST, Single task; DT, dual task; Seq, sequence; Rand, pseudorandom.

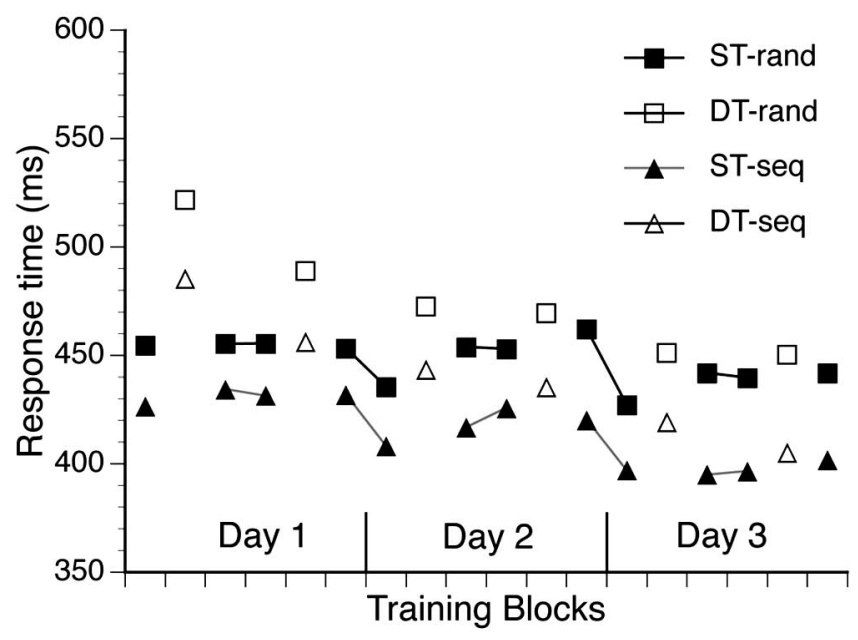

Figure 3. Response time data from training, separated by single-/dual-task and sequence/ random conditions. ST, Single task; DT, dual task; seq, sequence; rand, pseudorandom.

Region-of-interest (ROI) analyses were performed using the SPM ROI Toolbox (http://spm-toolbox.sourceforge.net). Regions were defined functionally based on voxelwise statistical maps, by growing a sphere (6 $\mathrm{mm}$ radius) around the local maxima in each cluster. For clusters of additional interest, the effect of each condition versus baseline was estimated for each subject by modeling the data using a finite impulse response model and averaging the response over the length of the trial block. This provided an estimate of the average signal change induced by each condition, without any assumptions regarding the shape of the response.

\section{Results}

\section{Behavioral results}

RTs obtained during scanning are presented in Figure 2, and those obtained during training are presented in Figure 3. Three subjects were excluded from the training analysis because of missing data in one session attributable to computer errors. All behavioral analyses were performed using repeated-measures ANOVA in SPSS (SPSS, Chicago, IL) with the Huyhn-Feldt correction for nonsphericity.
Scanning sessions

Subjects were highly accurate during scanning (mean accuracy $>95 \%$ in all blocks/conditions). Accuracy data for scanning are presented in supplemental Figure S1 (available at www. jneurosci.org as supplemental material). Scanning RT data were collapsed across single-task and dual-task blocks within each session (separately for sequence and pseudorandom blocks). The response time data were analyzed using a session (pre vs post) $\times$ condition (sequence vs pseudorandom) $\times$ task (single vs dual) repeated-measures ANOVA. There was a significant effect of session $\left(F_{(1,7)}=11.58 ; p=0.011\right)$, reflecting decreased response times overall from pretraining to posttraining, and an effect of condition $\left(F_{(1,7)}=17.876 ; p=0.004\right)$, reflecting faster responses to sequence versus pseudorandom blocks. There was also a significant session $\times$ condition interaction $\left(F_{(1,7)}=7.58 ; p=\right.$ $0.028)$, reflecting the greater decrease in response times for sequence versus pseudorandom blocks across scanning sessions. There was a significant effect of task $\left(F_{(1,7)}=13.38 ; p=0.008\right)$, reflecting slower responses on dual-task versus single-task blocks. There was a nonsignificant trend toward an interaction between task and session $\left(F_{(1,7)}=3.004 ; p=0.127\right)$. No other interactions were significant.

\section{Training sessions}

Subjects were highly accurate during training (mean accuracy $>95 \%$ in all blocks/conditions). Accuracy data for training are presented in supplemental Figure S2 (available at www. jneurosci.org as supplemental material). To perform a factorial ANOVA, the training response times were reduced by averaging across each pair of adjacent dual-task blocks and separately averaging across the two single-task blocks preceding those probe blocks and the two single-task blocks following the probe blocks. This was done separately for the dual-task probe blocks and the beginning and end of each training session (Fig. 1), giving two data points for each condition in each training session (and a total of six blocks across the three training sessions). The first and the last blocks were entered into a block $\times$ condition (sequence vs pseudorandom) $\times$ task (single vs dual) repeated-measures ANOVA. There was a main effect of block $\left(F_{(5,10)}=45.72 ; p<\right.$ $0.001)$, reflecting a downward trend in response times over training, as well as a main effect of condition $\left(F_{(1,10)}=33.93 ; p<\right.$ $0.001)$ and a main effect of task $\left(F_{(1,10)}=9.61 ; p=0.011\right)$. There was a significant task $\times$ block interaction $\left(F_{(5,10)}=11.53 ; p=\right.$ $0.007)$, reflecting a greater reduction in response times for dualtask compared with single-task blocks, consistent with the development of automaticity. The condition $\times$ block interaction was marginally significant $\left(F_{(5,10)}=3.097 ; p=0.109\right)$, suggesting that some sequence learning continued to occur during training. However, the condition $\times$ block $\times$ task interaction was not significant $\left(F_{(5,10)}=1.76 ; p=0.21\right)$, suggesting that the effect of the secondary task decreased similarly for sequence and pseudorandom conditions with practice.

Examination of dual-task costs showed a decrease from a mean cost of $61 \mathrm{~ms}$ ( $t$ test, $p=0.003)$ in the first training block versus a cost of $11 \mathrm{~ms}(p=0.362)$ in the final training block. The analogous costs during fMRI scanning were $53 \mathrm{~ms}$ pretraining $(t$ test, $p<0.001)$ versus $27 \mathrm{~ms}$ posttraining $(p=0.178)$. Thus, for both training and scanning, the dual-task effect was not significant after training, demonstrating the development of automaticity.

\section{Imaging results: whole-brain analyses}

Unless otherwise noted, all results were significant at $p<0.05$ (mapwise corrected using the cluster-based Gaussian random 


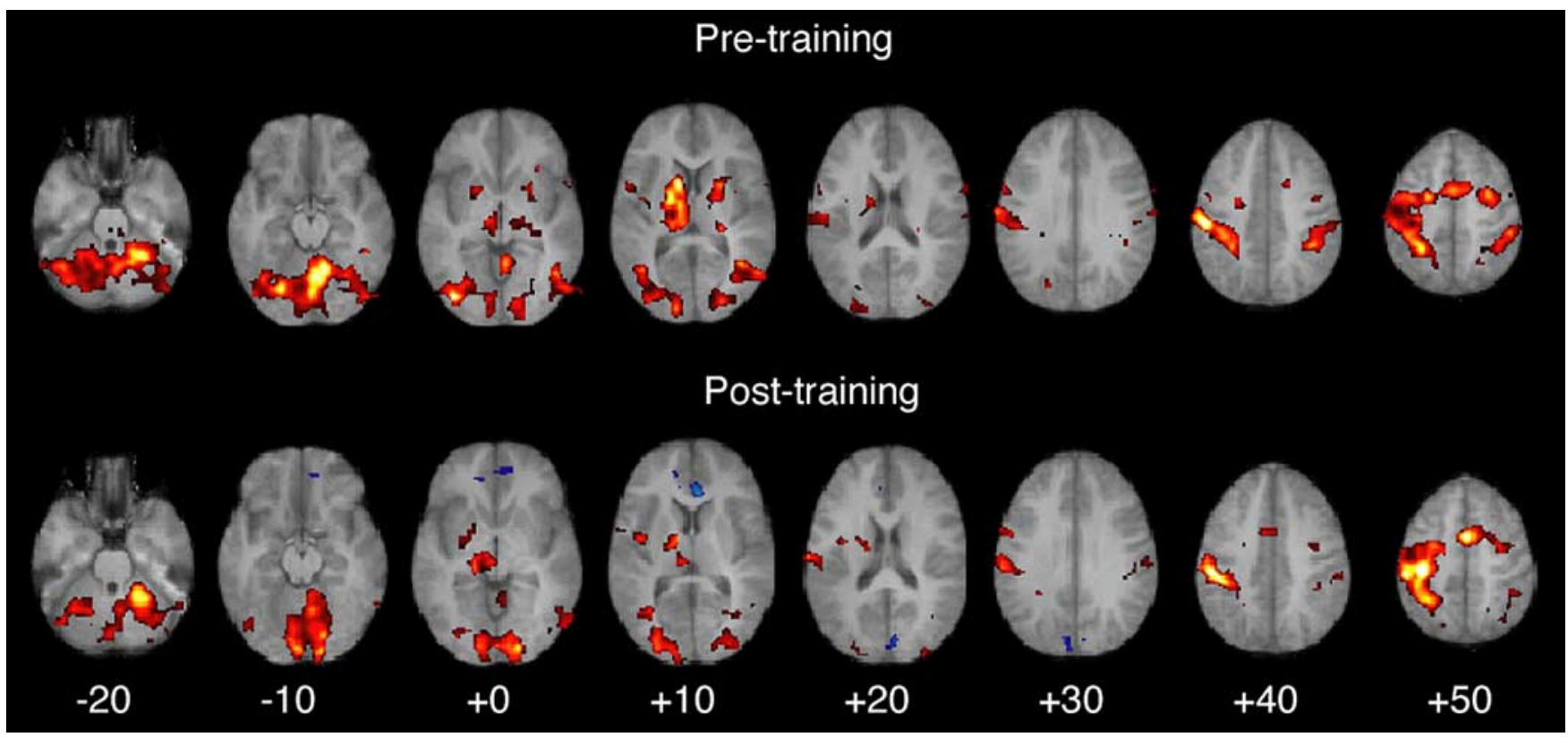

Figure 4. Thresholded statistical map for comparison of single-task condition (averaged over sequence and random blocks) versus rest ( $p<0.05$, cluster corrected for multiple comparisons). The top row presents results from pretraining session, and the bottom row presents posttraining results.

Table 1. Activation locations for comparison of single-task SRT versus rest (thresholded at $p<0.005$ uncorrected; cluster-level GRF correction, $p<0.05$ )

\begin{tabular}{|c|c|c|c|c|c|}
\hline Region & $x$ & $y$ & $z$ & Cluster extent (voxels) & $\operatorname{Max} T$ \\
\hline \multicolumn{6}{|l|}{ Pretraining } \\
\hline R inferior occipital/temporal & 18 & -54 & 15 & $8293^{*}$ & 11.34 \\
\hline L inferior occipital $^{a}$ & -42 & -81 & 0 & & 10.51 \\
\hline L inferior parietal $^{a}$ & -27 & -48 & 48 & & 9.37 \\
\hline L striatum $^{a}$ & -12 & 6 & 9 & & 9.33 \\
\hline R inferior occipital/temporal ${ }^{a}$ & 24 & -54 & -18 & & 9.09 \\
\hline R inferior parietal & 39 & -42 & 36 & 591 & 7.42 \\
\hline R inferior frontal & 60 & 3 & 15 & 105 & 4.46 \\
\hline \multicolumn{6}{|l|}{ Posttraining } \\
\hline L inferior parietal & -36 & -27 & 51 & 2245 & 11.72 \\
\hline $\mathrm{L}$ inferior parietal $^{a}$ & -48 & -30 & 42 & & 10.65 \\
\hline Medial frontal ${ }^{a}$ & 0 & -3 & 54 & & 9.47 \\
\hline R inferior occipital/temporal & 18 & -48 & -18 & 3529 & 8.40 \\
\hline L striatum & -12 & -6 & 9 & 490 & 6.81 \\
\hline R parietal & 39 & -45 & 51 & 297 & 4.88 \\
\hline
\end{tabular}

${ }^{a}$ For each cluster, all local maxima exceeding a voxel-level corrected $p<0.05$ threshold are presented.

GRF, Gaussian random field; $R$, right; $L$, left.

field correction in SPM99 after thresholding at $p<0.005$, uncorrected).

\section{Single-task activity}

Figure 4 presents a map of regions that were significantly different between single-task SRT performance (averaged over sequence and pseudorandom blocks) and rest; stereotactic locations for significant activations are listed in Table 1. Compared with rest, performance was associated with bilateral activity in occipital/temporal cortex, sensorimotor and dorsal premotor (PMd) cortex, supplementary motor area (SMA), striatum (caudate/putamen), and cerebellar cortex and deep nuclei. Activity was also compared between pretraining and posttraining sessions to identify learning-related changes. There were no changes at a corrected threshold. Because of the a priori hypothesis of changes in basal ganglia activation based on previous studies, activity was examined within a broad basal ganglia ROI derived from the
Automated Anatomical Labeling Atlas (Tzourio-Mazoyer et al., 2002) using small volume correction (SVC) within SPM99. Within this region, there was a significant decrease in basal ganglia activation from pretraining to posttraining, corrected for the extent of the ROI.

Sequence-related activity

Activity was compared between sequence and pseudorandom conditions separately for single-task performance in each session. In the pretraining session, there were no differences in either direction that survived a corrected threshold. However, there was marginally greater activity for sequence compared with pseudorandom blocks in the right caudate nucleus ( $p=$ 0.06 , corrected), and this activation was significant in an SVC analysis using the basal ganglia ROI described above. In the posttraining scan, there were no regions with significantly greater activity for sequence compared with pseudorandom conditions, and an SVC analysis of the basal ganglia was not significant. However, several regions showed greater activity for pseudorandom compared with sequence blocks, including left medial intraparietal cortex and left inferior frontal gyrus (IFG); a previous study by Doyon et al. (1996) found similar increases for novel sequence versus an overtrained sequence, although in that study, the differences were right lateralized. An additional analysis compared sequence and pseudorandom conditions under dual-task conditions. In the pretraining session, there were no significant differences at the whole-brain level. After training, there were no regions with activity for the sequence $>$ random comparison, but the left putamen and left premotor cortex showed significantly greater activity for pseudorandom than sequence conditions. 


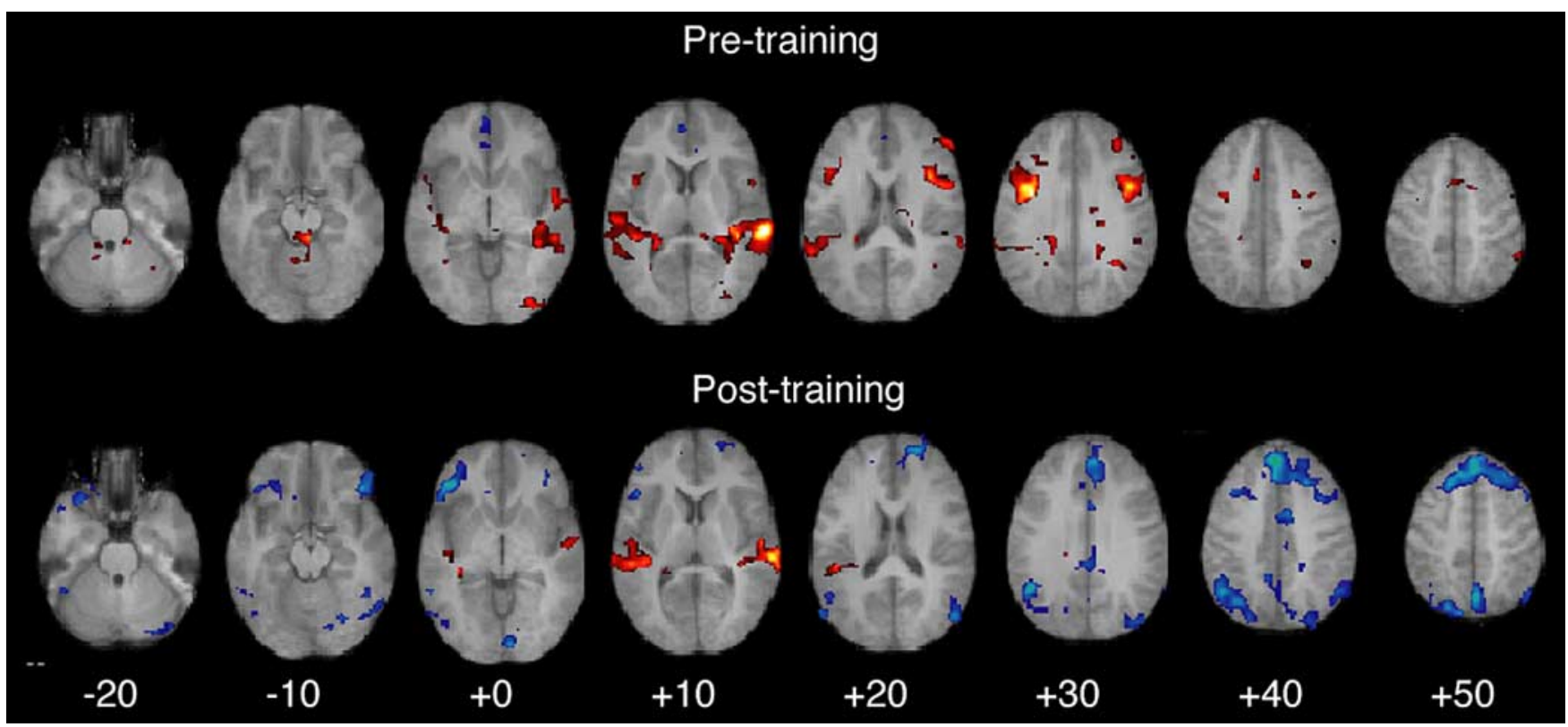

Figure 5. Thresholded statistical map for comparison of single-task versus dual-task conditions (averaged over sequence and random conditions) ( $p<0.05$, cluster corrected for multiple comparisons). The top row presents results from pretraining session, and the bottom row presents posttraining results.

\section{Dual-task effects}

Comparisons of single-task versus dualtask performance (averaged across sequence and random blocks) are presented in Figure 5 and Table 2. Activity during dual-task performance was compared with single-task performance separately in each session. In the pretraining session, an extensive set of regions were more active during dual-task compared with singletask performance, including bilateral auditory cortices, bilateral lateral premotor cortex, pre-SMA, and right caudate body. In the posttraining session, the only regions showing significantly more activity for dual- versus single-task conditions were bilateral auditory cortices. Direct comparison of dual-task activation between pretraining and posttraining sessions identified decreases in right lateral (inferior/middle) prefrontal, premotor, and bilateral parietal cortex, as well as the cerebellum (Fig. 6). Additional analyses were performed to determine whether the single versus dual effect differed between sequence and random conditions; no significant differences were found for either pretraining or posttraining, suggesting that the dual-task effect was qualitatively similar between these conditions.

\section{Imaging results: region of interest analyses}

To further characterize activity in the regions identified in the foregoing whole-brain analyses, ROI analyses were performed on a number of regions of particular interest identified in the foregoing analyses; the results of these analyses are presented in Figure 7. For each ROI, a two (pre/post) $\times$ two (single/dual task) $\times$ two (sequence/pseudorandom) repeated-measures ANOVA was performed on the extracted mean signal across the ROI. Results of these ANOVAs are presented in Table 3.
Table 2. Activation locations for comparison of dual-task SRT versus single-task SRT (thresholded at $p<0.005$, uncorrected; cluster-level GRF correction, $p<0.05$ )

\begin{tabular}{|c|c|c|c|c|c|}
\hline Region & $x$ & $y$ & $z$ & Cluster extent (voxels) & $\operatorname{Max} T$ \\
\hline \multicolumn{6}{|l|}{ Pretraining } \\
\hline R superior temporal & 60 & -30 & 6 & 2249 & 10.59 \\
\hline R superior temporal ${ }^{a}$ & 66 & -30 & 12 & & 9.01 \\
\hline L inferior frontal & -36 & 0 & 27 & 503 & 10.35 \\
\hline Inferior colliculus & 6 & -36 & -12 & 141 & 6.46 \\
\hline Medial cerebellum & 3 & -78 & -27 & 284 & 6.04 \\
\hline L superior temporal & -42 & -51 & 6 & 953 & 6.03 \\
\hline R occipital & 27 & -84 & 6 & 121 & 5.41 \\
\hline \multicolumn{6}{|l|}{ Posttraining } \\
\hline L superior temporal & -54 & -33 & 12 & 471 & 9.07 \\
\hline R superior temporal & 63 & -30 & 12 & 285 & 8.79 \\
\hline \multicolumn{6}{|l|}{ Pre versus Post } \\
\hline L inferior/middle frontal & -42 & 9 & 30 & 741 & 9.33 \\
\hline Roccipital & 33 & -81 & 12 & 1129 & 7.42 \\
\hline Loccipital & -27 & -78 & 12 & 476 & 6.75 \\
\hline R inferior/middle frontal & 36 & 30 & 42 & 691 & 6.71 \\
\hline R superior parietal & 30 & -78 & 45 & 530 & 6.37 \\
\hline L superior parietal & -18 & -75 & 51 & 390 & 6.13 \\
\hline Medial parietal/precuneus & 6 & -66 & 51 & 113 & 5.46 \\
\hline
\end{tabular}

${ }^{a}$ For each cluster, all local maxima exceeding a voxel-level corrected $p<0.05$ threshold are presented. GRF, Gaussian random field; $R$, right; L, left.

Left putamen/globus pallidus

This region showed an interaction between pretraining/posttraining and sequence type; signal decreased from pretraining to posttraining for sequence conditions but remained constant for random conditions. This effect was equivalent between singletask and dual-task performance.

\section{SMA}

There were no significant effects in the SMA region; however, there was a trend toward a training $\times$ sequence type interaction, with a pattern similar to the left putamen/pallidum region.

\section{PMd}

Left and right PMd exhibited markedly different responses. Left PMd activity was quite consistent across all conditions, with no 


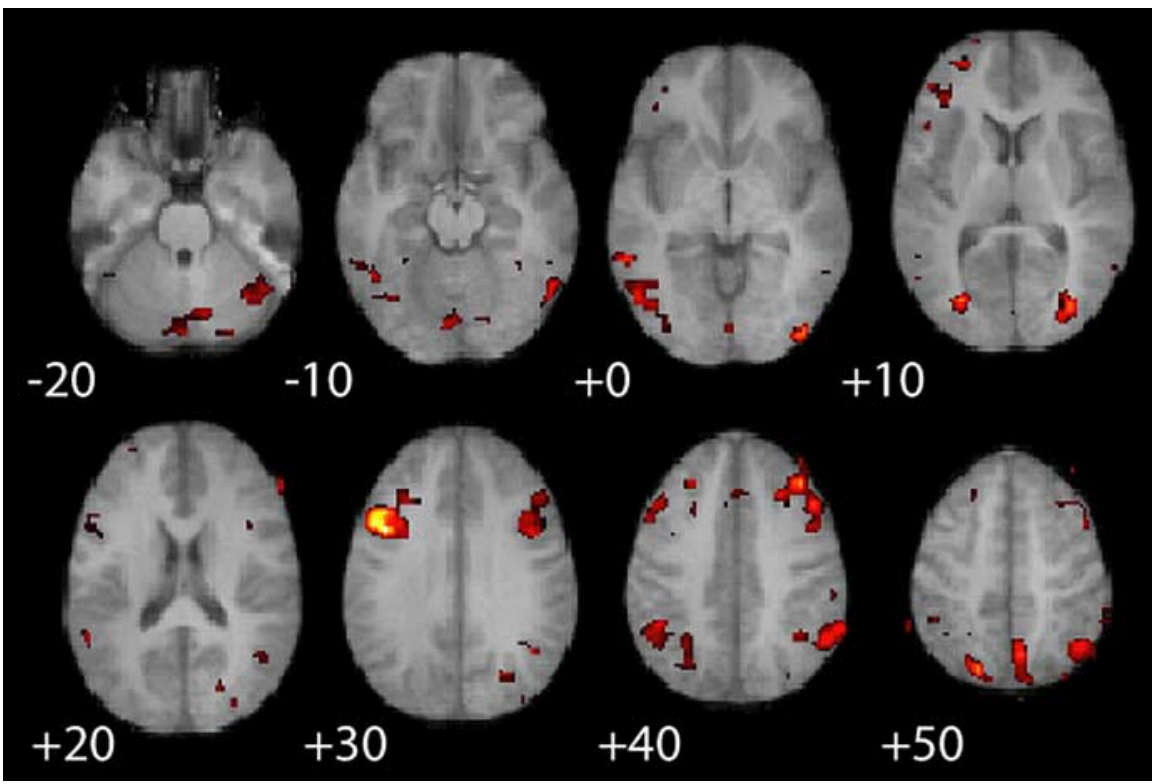

Figure 6. Thresholded statistical map for interaction between pretraining/posttraining and single/dual task $(p<0.05$, cluster corrected for multiple comparisons).

significant effects in the ANOVA. Right PMd, in contrast, exhibited substantial decreases in signal from pretraining to posttraining, except in the single-task pseudorandom condition in which signal was constant. This was reflected in a significant three-way interaction, as well as a significant pretraining/posttraining $\times$ single-/dual-task interaction. This difference in response between left and right PMd was confirmed in a four-way (ROI $\times$ pre/post $\times$ task $\times$ sequence) ANOVA; the four-way interaction was significant ( $p=0.025)$.

\section{Ventral premotor cortex/inferior frontal gyrus}

A region encompassing the ventral premotor cortex (PMv) and inferior prefrontal gyrus showed a bilateral pattern of activity different from those observed in PMd. Most strikingly, PMv/IFG was only active during dual-task conditions in the pretraining session. For both left and right PMv/IFG regions, there was a significant main effect of single/dual task and a significant pre/ post $\times$ single-/dual-task interaction. Comparison between left and right PMv/IFG regions in a four-way ANOVA revealed no significant interactions with hemisphere, suggesting relatively similar patterns of response across hemispheres.

\section{Right dorsolateral prefrontal cortex}

Right dorsolateral prefrontal cortex (DLPFC) showed a pattern very similar to PMv/IFG, with activity only during dual-task conditions. The main effect of single/dual task as well as the pre/ post $\times$ single-/dual-task interaction were both significant.

\section{Right caudate body}

The right body of the caudate nucleus showed a pattern very similar to $\mathrm{PMv} / \mathrm{IFG}$ and right DLPFC, with a significant pre/ post $X$ single-/dual-task interaction.

\section{Discussion}

The present study examined changes in neural activity associated with the acquisition of automaticity in a motor learning task. There were three major findings. First, novice performance of an SRT task with a concurrent working memory load resulted in activation of a network, including PMv/IFG and DLPFC and right caudate body, compared with performance without a con- current task. This suggests that these regions are critical for cognitive control during early learning. Other regions, including dorsal premotor cortex and SMA, were equally active regardless of the presence of the secondary task, consistent with a more fundamental role in motor programming. Second, practice was associated with the development of automaticity (as indexed by the elimination of dualtask interference), and this change was associated with decreased activation in PMv/IFG, DLPFC, and right caudate regions. Finally, basal ganglia regions [including putamen, globus pallidus (GP), and caudate head] exhibited an interaction of sequence type and time, such that training resulted in decreased activity for sequential trials but not for pseudorandom trials. These results provide direct evidence that these regions are involved in the acquisition of motor sequence knowledge, consistent with previous imaging (Grafton et al., 1995; Rauch et al., 1997) and neuropsychological (Knopman and Nissen, 1991; Willingham and Koroshetz, 1993) results.

It is of note that the main findings regarding automaticity in this study were general to both sequence and random conditions in the SRT task. This is supported by the lack of an interaction between dual-task effects and sequence versus random conditions on response time during training and by the lack of differences in the neural signature of dual-task effects for sequence versus random conditions. These results diverge from previous behavioral studies, which found that dual-task conditions resulted in impaired acquisition (Curran and Keele, 1993) and/or expression (Frensch et al., 1998) of sequence knowledge. It is possible that this difference from previous results arose from a procedural difference between the present study and those previous studies. Nearly all previous studies presented the tone at randomly spaced times after presentation of the SRT stimulus and approximately coincident with the motor response or in the interval between the motor response and the next stimulus (cf. Hsiao and Reber, 2001), whereas we presented the tone concurrently with the onset of the SRT stimulus. Hsiao and Reber (2001) systematically varied the occurrence of the tone within the response-stimulus interval and found the greatest interference late in this interval. This suggests that a consistently timed tone before the response may have reduced the disruption of sequence timing. Instead, the presence of the tone appeared to exert a general effect on motor responding during earlier sessions.

Several previous studies have examined neural changes associated with extensive (multisession) training on SRT tasks. Karni et al. (1995) trained subjects on an explicit five-element finger movement sequence for 10-20 min of practice per day for 5 weeks. No secondary task was performed, but the fact that subjects continued to practice for 2 weeks beyond asymptote suggests that automaticity was likely achieved. This study used a surface coil and only imaged a small region of motor cortex, in which an increase in activity related to sequence learning was observed compared with an untrained motor sequence. No increase was observed in motor cortex in the present study, but this may have been attributable to the degree of smoothing applied to the data, which may have obscured small, localized changes in activity. $\mathrm{Wu}$ 
A) L Putamen/GP

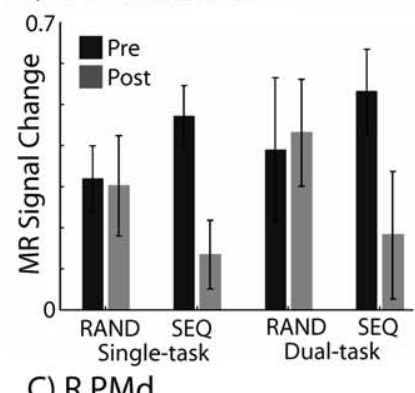

C) R PMd

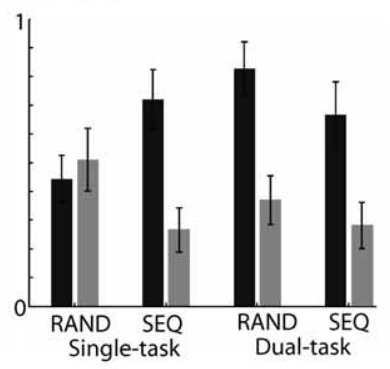

E) LPMv/IFG

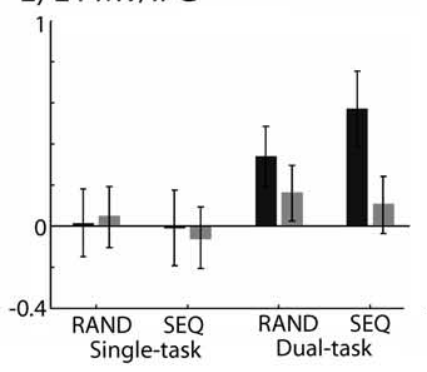

G) R MFG

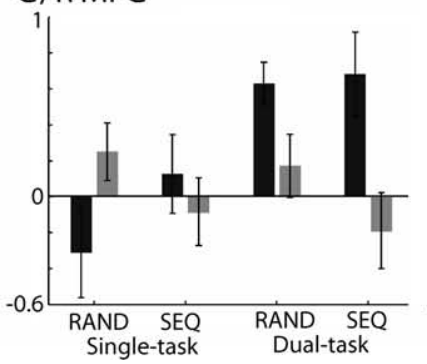

B) SMA

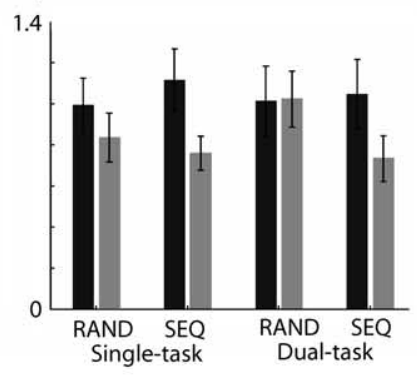

D) L PMd

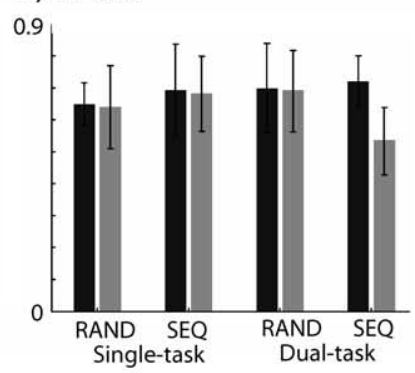

F) R PMv/IFG

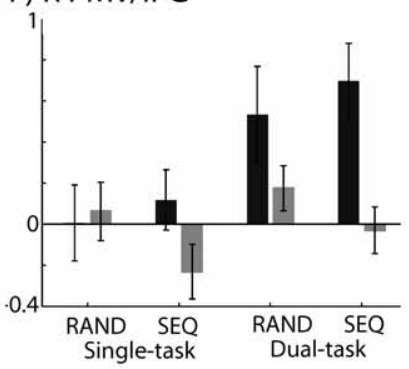

H) R caudate body 0.7

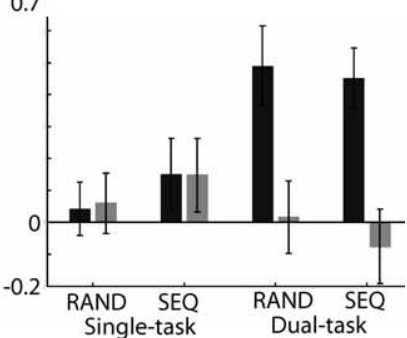

Figure 7. Bar graphs of signal extracted from regions of interest, with SE. Statistical analyses of these data and stereotactic locations for each region are presented in Table 3. L, Left; R, right; MFG, middle frontal gyrus; SEQ, sequence; RAND, pseudorandom.

et al. (2004) scanned before and after an explicit sequence learning paradigm presenting two sequences of different lengths to each subject ( 4 or 12 elements) and administering a secondary task to index the degree of automaticity. Automaticity was achieved within $2-4 \mathrm{~h}$ of training on both sequences. A number of regions showed decreased activation after training, including bilateral prefrontal and parietal regions, cerebellum, and caudate nucleus; as in the present study, no increases in activation were observed after training. However, this study did not directly image SRT performance under dual-task conditions. Thus, our results extend previous findings by demonstrating that automaticity is associated with specific decreases in activation in a frontostriatal network during dual-task performance.

Although the focus of our study was on motor skill learning in general, there were some modest differences in activity between sequence and pseudorandom conditions. Several previous studies have found patterns of sequence-specific activation in the SRT that differ depending on whether sequence learning was implicit or explicit (Grafton et al., 1995; Rauch et al., 1995; Hazeltine et al., 1997; Honda et al., 1998). In the present study, awareness of the repeated sequence was not assessed. The second-order conditional sequence used in the present study and the mixing of sequence and random blocks was likely to have reduced sequence awareness, although it is possible that some subjects acquired at least partial explicit sequence knowledge. Our neuroimaging results overlap to some extent with previous studies of implicit sequence learning, in that there was a strong trend for sequencespecific caudate activation early in training. It is unclear whether the possibility of explicit sequence knowledge in our subjects had a major impact on the pattern of results, given more recent findings of Willingham et al. (2002) that regions active during implicit learning were also active during explicit learning (but not vice versa).

Because the development of automaticity in this task was not sequence specific, it must reflect changes in the processing of more basic invariant features of the tasks that may be shared by other motor skill tasks. One major aspect of task-general learning in our paradigm may be the tuning of spatially compatible stimulus-response mappings. In this context, it is interesting that previous studies have associated ventrolateral prefrontal cortex with the learning, maintenance, and controlled retrieval of stimulusresponse associations (Asaad et al., 1998; Passingham et al., 2000; Bunge et al., 2003). The increased activity of bilateral ventrolateral prefrontal cortex (PMv/IFG) in our dual-task condition before training suggests that dual-task conditions may require greater control over stimulus-response mapping rules, even in cases in which the mappings are spatially compatible. With extended practice, these mappings appear to be retrieved automatically and thus independently of ventrolateral prefrontal circuitry.

Automaticity, cognitive control, and cortico-striatal systems The present results suggest that a cortico-striatal network involving the lateral prefrontal cortex and caudate nucleus is primarily responsible for the engagement of cognitive control during dualtask performance. These regions exhibit activity during novice dual-task performance, which decreases after the development of automaticity on the SRT task. This finding is consistent with the results from a growing body of studies implicating the prefrontal cortex in cognitive control (cf. Miller and Cohen, 2001; Ridderinkhof et al., 2004) and also with the findings of previous studies of motor skill acquisition (Jenkins et al., 1994; Jueptner et al., 1997a; Toni et al., 1998). The specific control operations implemented by these regions remain unclear. Because dual-task activity in prefrontal cortex declined with training although working memory requirements remained constant (as did working memory performance), it seems unlikely that the premotor and lateral prefrontal cortical activations observed here reflect working memory maintenance per se. Alternatively, the lateral prefrontal cortex may be involved in coordination of switching between the two tasks, perhaps involving shifting of attention to different task characteristics (Jiang, 2004) or the detection of salient stimuli across modalities (Corbetta and Shulman, 2002). The right inferior prefrontal cortex may play a particular role in the inhibition of competing task sets related to its more general role in response inhibition (Aron et al., 2004).

There is substantial debate regarding the cognitive processes 
Table 3. Results from two (pre/post) $\times$ two (single/dual) $\times$ two (sequence/random) repeated-measures ANOVA on MR signal extracted from selected regions of interest displayed in Figure 7

\begin{tabular}{|c|c|c|c|c|c|c|c|c|c|c|}
\hline Location & $x$ & $y$ & $z$ & Pre/post & Task & Sequence & $\begin{array}{l}\text { Pre/post } \times \\
\text { task }\end{array}$ & $\begin{array}{l}\text { Pre/post } X \\
\text { sequence }\end{array}$ & $\begin{array}{l}\text { Task } \times \\
\text { sequence }\end{array}$ & $\begin{array}{l}\text { Pre/post } \times \text { task } \times \\
\text { sequence }\end{array}$ \\
\hline$A, \mathrm{~L}$ putamen/GP $(n=14)$ & -18 & -6 & 12 & 0.109 & & & & 0.031 & & \\
\hline$B$, Supplementary motor area $(n=13)$ & 0 & -3 & 54 & & & & & 0.140 & & \\
\hline C, R dorsal premotor $(n=13)$ & 27 & -3 & 54 & 0.003 & & & 0.025 & 0.097 & & 0.022 \\
\hline$D, \mathrm{~L}$ dorsal premotor $(n=13)$ & -24 & -9 & 54 & & & & & & & \\
\hline$E, L$ ventral premotor/inferior frontal $(n=14)$ & -36 & 0 & 27 & & $<0.001$ & & 0.005 & 0.136 & 0.141 & \\
\hline$F$, R ventral premotor/inferior frontal $(n=14)$ & 45 & 6 & 30 & 0.065 & 0.002 & & 0.007 & 0.048 & & \\
\hline$G, R$ middle frontal $(n=14)$ & 33 & 39 & 30 & & 0.013 & & 0.012 & 0.019 & & \\
\hline$H, R$ caudate body $(n=13)$ & 15 & -9 & 24 & 0.022 & 0.099 & & 0.002 & & 0.136 & \\
\hline
\end{tabular}

Lettered locations correspond to those presented in Figure 7. $p$ values are presented for all results with $p<0.15$; blank cells represent nonsignificant ( $p>0.15)$ results. The number of subjects entering the analysis is listed for each region; data from one subject were missing for several regions because the signal in those regions failed to meet a minimal omnibus $F$ threshold in the single-subject analysis (from which the ROI data are estimated).

that underlie dual-task interference and the development of automaticity. Pashler et al. (2001) have argued that behavioral interference attributable to a secondary task is caused by interference at the stage of response selection and that this response selection bottleneck is not eliminated even after automaticity is achieved, although its effects may be minimized under particular task conditions. Schumacher et al. (2001), however, have argued that response selection bottlenecks can be eliminated with practice. The implications of the present results for this debate depend on how prefrontal activation during early dual-task performance is interpreted. If DLPFC/PMv activation reflects processes involved in resolving response selection conflicts, then the elimination of this activation by the end of training would suggest that response selection conflicts are no longer evident after development of automaticity. In this context, it is interesting to note that the PMv and middle frontal gyrus regions observed in the present study are similar to regions observed in relation to response selection by Bunge et al. (2003) and Schumacher and D'Esposito (2002). However, resolution of this question would require conjoint examination of automaticity and response selection manipulations. By identifying brain regions involved in response selection and by examining activity in these regions as performance becomes automatic with practice, it may be possible to resolve the role of response selection in the development of automaticity.

\section{The striatum and sequence learning}

Although robust significant differences between sequence and random conditions were not evident at the whole-brain level, our ROI analyses primarily found decreases in basal ganglia regions related to sequence acquisition, whereas previous studies have primarily found increases. Several differences between the current and previous studies may account for this. In the current study, participants underwent $3 \mathrm{~h}$ of training, allowing them to reach automatic performance levels, whereas previous studies have provided much less training. It has been suggested that the basal ganglia are important for chunking action sequences (Graybiel, 1998). Chunking allows performance of a well learned motor sequence to be executed as a single unit of activity rather than multiple individual actions. Thus, decreased activity in the basal ganglia may reflect a more effective (chunked) representation that accompanies automatization. Several other studies that have found increases in the basal ganglia examined explicit sequence learning (Seitz et al., 1990; Seitz and Roland, 1992; Schlaug et al., 1994) or compared pretrained explicitly learned sequences with learning of new sequences (Jenkins et al., 1994). The present study, conversely, used an incidental learning paradigm, but it is not known whether subjects may have also acquired explicit sequence knowledge during training.
The caudate and putamen/GP exhibited very different patterns of behavior in the present study. The putamen was active early and decreased with training for sequence but not pseudorandom blocks, whereas the caudate body was active only in response to dual-task conditions before training. Previous studies have also found dissociations between these regions; in particular, it has been suggested that the putamen/GP is primarily involved in performance of motor skills, whereas the caudate is involved in acquiring the motor sequence knowledge (Jueptner et al., 1997b). Consistent with this argument, there was a trend toward greater activity in the caudate for sequence versus pseudorandom conditions during the pretraining scan. Other studies using incidental learning have found increases in putamen but also found decreases in the caudate with skill development (Grafton et al., 1995). The results of our study suggest that, whereas putamen activity may increase during early portions of skill acquisition (as measured in these previous studies), it decreases with the development of automaticity. These results highlight the usefulness of examining performance after extensive training to fully characterize the time course of activity.

\section{References}

Aron AR, Robbins TW, Poldrack RA (2004) Inhibition and the right inferior frontal cortex. Trends Cogn Sci 8:170-177.

Asaad WF, Rainer G, Miller EK (1998) Neural activity in the primate prefrontal cortex during associative learning. Neuron 21:1399-1407.

Bunge SA, Kahn I, Wallis JD, Miller EK, Wagner AD (2003) Neural circuits subserving the retrieval and maintenance of abstract rules. J Neurophysiol 90:3419-3428.

Corbetta M, Shulman GL (2002) Control of goal-directed and stimulusdriven attention in the brain. Nat Rev Neurosci 3:201-215.

Curran T, Keele SW (1993) Attentional and nonattentional forms of sequence learning. J Exp Psychol Learn Mem Cogn 19:189-202.

Doyon J, Owen AM, Petrides M, Sziklas V, Evans AC (1996) Functional anatomy of visuomotor skill learning in human subjects examined with positron emission tomography. Eur J Neurosci 8:637-648.

Dreher JC, Grafman J (2003) Dissociating the roles of the rostral anterior cingulate and the lateral prefrontal cortices in performing two tasks simultaneously or successively. Cereb Cortex 13:329-339.

Frensch PA, Lin J, Buchner A (1998) Learning versus behavioral expression of the learned: the effects of a secondary tone-counting task on implicit learning in the serial reaction task. Psychol Forsch 61:83-98.

Grafton ST, Woods RP, Tyszka M (1994) Functional imaging of procedural motor learning: relating cerebral blood flow with individual subject performance. Hum Brain Mapp 1:221-234.

Grafton ST, Hazeltine E, Ivry R (1995) Functional mapping of sequence learning in normal humans. J Cogn Neurosci 7:497-510.

Graybiel AM (1998) The basal ganglia and chunking of action repertoires. Neurobiol Learn Mem 70:119-136.

Hazeltine E, Grafton ST, Ivry R (1997) Attention and stimulus characteristic determine the locus of motor-sequence encoding. A PET study. Brain 120:123-140. 
Honda M, Deiber MP, Ibanez V, Pascual-Leone A, Zhuang P, Hallett M (1998) Dynamic cortical involvement in implicit and explicit motor sequence learning. A PET study. Brain 121:2159-2173.

Hsiao AT, Reber AS (2001) The dual-task SRT procedure: fine-tuning the timing. Psychon Bull Rev 8:336-342.

James W (1890) Principles of psychology. New York: Holt.

Jenkins IH, Brooks DJ, Nixon PD, Frackowiak RS, Passingham RE (1994) Motor sequence learning: a study with positron emission tomography. J Neurosci 14:3775-3790.

Jiang Y (2004) Resolving dual-task interference: an fMRI study. NeuroImage 22:748-754.

Jueptner M, Stephan KM, Frith CD, Brooks DJ, Frackowiak RS, Passingham RE (1997a) Anatomy of motor learning. I. Frontal cortex and attention to action. J Neurophysiol 77:1313-1324.

Jueptner M, Frith CD, Brooks DJ, Frackowiak RS, Passingham RE (1997b) Anatomy of motor learning. II. Subcortical structures and learning by trial and error. J Neurophysiol 77:1325-1337.

Karni A, Meyer G, Jezzard P, Adams MM, Turner R, Ungerleider LG (1995) Functional MRI evidence for adult motor cortex plasticity during motor skill learning. Nature 377:155-158.

Knopman D, Nissen MJ (1991) Procedural learning is impaired in Huntington's disease: evidence from the serial reaction time task. Neuropsychologia 29:245-254.

Logan GD (1979) On the use of a concurrent memory load to measure attention and automaticity. J Exp Psychol Hum Percept Perform 5:189-207.

Miller EK, Cohen JD (2001) An integrative theory of prefrontal cortex function. Annu Rev Neurosci 24:167-202.

Nissen MJ, Bullemer P (1987) Attentional requirements of learning: evidence from performance measures. Cognit Psychol 19:1-32.

Norman DA, Shallice T (1986) Attention to action: willed and automatic control of behavior. In: Consciousness and self-regulation (Davidson RJ, Schwartz GE, Shapiro D, eds). New York: Plenum.

Pashler H, Johnston JC, Ruthruff E (2001) Attention and performance. Annu Rev Psychol 52:629-651.

Passingham RE, Toni I, Rushworth MF (2000) Specialisation within the prefrontal cortex: the ventral prefrontal cortex and associative learning. Exp Brain Res 133:103-113.

Penhune VB, Doyon J (2002) Dynamic cortical and subcortical networks in learning and delayed recall of timed motor sequences. J Neurosci 22:1397-1406.

Perruchet P, Amorim MA (1992) Conscious knowledge and changes in performance in sequence learning: evidence against dissociation. J Exp Psychol Learn Mem Cogn 18:785-800.

Poldrack RA, Gabrieli JD (2001) Characterizing the neural mechanisms of skill learning and repetition priming: evidence from mirror reading. Brain 124:67-82.

Poldrack RA, Desmond JE, Glover GH, Gabrieli JDE (1998) The neural basis of visual skill learning: an fMRI study of mirror-reading. Cereb Cortex 8:1-10.

Posner MI, Snyder CRR (1975) Attention and cognitive control. In: Information processing and cognition: The Loyola Symposium (Solso RL, ed), pp 55-85. Hillsdale, NJ: Erlbaum.
Rauch S, Savage C, Brown H, Curran T, Alpert N, Kendrick A, Fischman A, Kosslyn SM (1995) A PET investigation of implicit and explicit sequence learning. Hum Brain Mapp 3:271-286.

Rauch SL, Whalen PJ, Savage CR, Curran T, Kendrick A, Brown HD, Bush G, Breiter HC, Rosen BR (1997) Striatal recruitment during an implicit sequence learning task as measured by functional magnetic resonance imaging. Hum Brain Mapp 5:124-132.

Reed J, Johnson P (1994) Assessing implicit learning with indirect tests: determining what is learnt about sequence structure. J Exp Psychol Learn Mem Cogn 20:585-594.

Ridderinkhof KR, Ullsperger M, Crone EA, Nieuwenhuis S (2004) The role of the medial frontal cortex in cognitive control. Science 306:443-447.

Schlaug G, Knorr U, Seitz RJ (1994) Inter-subject variability of cerebral activations in acquiring a motor skill: a study with positron emission tomography. Exp Brain Res 98:523-534.

Schumacher EH, D’Esposito M (2002) Neural implementation of response selection in humans as revealed by localized effects of stimulus-response compatibility on brain activation. Hum Brain Mapp 17:193-201.

Schumacher EH, Seymour TL, Glass JM, Fencsik DE, Lauber EJ, Kieras DE, Meyer DE (2001) Virtually perfect time sharing in dual-task performance: uncorking the central cognitive bottleneck. Psychol Sci 12:101-108.

Seitz RJ, Roland PE (1992) Learning of sequential finger movements in man: a combined kinematic and positron emission tomography (PET) study. Eur J Neurosci 4:154-165.

Seitz RJ, Roland PE, Bohm C, Greitz T, Stone-Elander S (1990) Motor learning in man: a positron emission tomographic study. NeuroReport $1: 57-60$.

Sohn MH, Ursu S, Anderson JR, Stenger VA, Carter CS (2000) Inaugural article: the role of prefrontal cortex and posterior parietal cortex in task switching. Proc Natl Acad Sci USA 97:13448-13453.

Toni I, Krams M, Turner R, Passingham RE (1998) The time course of changes during motor sequence learning: a whole-brain fMRI study. NeuroImage 8:50-61.

Tzourio-Mazoyer N, Landeau B, Papathanassiou D, Crivello F, Etard O, Delcroix N, Mazoyer B, Joliot M (2002) Automated anatomical labeling of activations in SPM using a macroscopic anatomical parcellation of the MNI MRI single-subject brain. NeuroImage 15:273-289.

Van Horn JD, Gold JM, Esposito G, Ostrem JL, Mattay V, Weinberger DR, Berman KF (1998) Changing patterns of brain activation during maze learning. Brain Res 793:29-38.

Willingham DB, Koroshetz WJ (1993) Evidence for dissociable motor skills in Huntington's disease patients. Psychobiology 21:173-182.

Willingham DB, Nissen MJ, Bullemer P (1989) On the development of procedural knowledge. J Exp Psychol Learn Mem Cogn 15:1047-1060.

Willingham DB, Salidis J, Gabrieli JD (2002) Direct comparison of neural systems mediating conscious and unconscious skill learning. J Neurophysiol 88:1451-1460.

Wu T, Kansaku K, Hallett M (2004) How self-initiated memorized movements become automatic: a functional MRI study. J Neurophysiol 91:1690-1698.

Wylie GR, Javitt DC, Foxe JJ (2003) Cognitive control processes during an anticipated switch of task. Eur J Neurosci 17:667-672. 